Honam Mathematical J. 36 (2014), No. 1, pp. 103-112

http://dx.doi.org/10.5831/HMJ.2014.36.1.103

\title{
THE REPRESENTATION OF THE GOLDEN RATIO BY THE CONTINUED FRACTION
}

\author{
Seung Soo Kim*, Mi Yeon Ko, and Yong Hun Lee
}

\begin{abstract}
There are several theories to say that 'Mathematics is beautiful', but the typical one of them is a theory about the golden ratio. Often the golden ratio apt to be considered only as the geometric shapes or the simple number of ratio used in buildings and arts. However in this paper, we studied to consider the mathematical theories which are contained in their inside. In particular, we investigate the various expressions of the continued fraction which are represented by the golden ratio.
\end{abstract}

\section{Introduction}

First, we introduce the well known expression of the continued fraction from the definition of golden ratio as follows.

$$
\phi=1+\frac{1}{1+\frac{1}{1+\frac{1}{1+\frac{1}{}}}} .
$$

As is well known, we say that two quantities are in the golden ratio if the ratio of the sum of the quantities to the larger quantity is equal to the ratio of the larger quantity to the smaller one.

Let $a$ and $b$ be the two quantities satisfying the golden ratio with $a>b$. By definition of the golden ratio, two ratio $\frac{a+b}{a}$ and $\frac{a}{b}$ are equal to the golden ratio $\phi$. That is,

$$
\frac{a+b}{a}=\frac{a}{b}=\phi .
$$

Received December 2, 2013. Accepted January 15, 2014.

2010 Mathematics Subject Classification. 76S05, 65C20; 80A20, 74G15.

Key words and phrases. golden ratio, continued fraction, iterated fixed point theorem.

${ }^{*}$ Corresponding author 
Hence the golden ratio $\phi$ satisfies the equation

$$
\phi=\frac{a+b}{a}=1+\frac{b}{a}=1+\frac{1}{\phi} .
$$

By multiplying $\phi$ on the both sides of equation (1.3), we give the equation

$$
\phi^{2}=\phi+1 \text {. }
$$

Using this quadratic formula (1.4), we obtain the only positive solution as

$$
\phi=\frac{1+\sqrt{5}}{2}=1.6180339887 \cdots .
$$

From the equation (1.3), the golden ratio $\phi$ also satisfies $\phi-1=\frac{1}{\phi}$.

The golden ratio can be expressed by continued fraction. From the equation $\phi=1+\frac{1}{\phi}$ and once again substituting this expression into the $\phi$ of right-hand side, then we have $\phi=1+\frac{1}{1+\frac{1}{\phi}}$. If you continue to repeat, it can be expressed continued fraction as the equation (1.1).

However, it is not clear mathematically. We looked for the mathematical theory to clearly explain the mathematical meaning of '...' in the above representation. For this purpose, we construct the sequence which converges to the golden ratio by fixed point iteration method.

This paper is consists as following: Chapter 2 explain about definition of the golden ratio and the expression of the continued fraction of the various representations. We also introduce about the fixed point theorem that the functions which satisfy some conditions have only one fixed point. In chapter 3 , we introduce about the fixed point iteration that the sequence founded from the function that satisfy the condition of the fixed point theorem converges to fixed point for the appropriate initial value. We find some example sequences through fixed point iteration converges to fixed point by apply the expression of continued fraction of the golden ratio. Finally in chapter 4, we calculated by the MS-EXCEL program for functions that intuitively and theoretically converges to golden ratio. And we reported the practical values of the sequence and the rate of convergence through the fixed point iteration.

\section{Preliminaries}

In this section, we introduce some definitions and the basic facts about the golden ratio and the fixed point iteration method. 
Now let's look at the method of another way to find the expression of the continued fraction. The golden ratio is the root of the quadratic equation

$$
x^{2}-x-1=0 .
$$

To obtain the root $x=c$ of the equation $f(x)=0$, which can be transformed into $g(x)-x=0$, define the sequence $\left\{x_{n}\right\}$ by $x_{n+1}=g\left(x_{n}\right)$, $n \geq 0$ with initial guess $x_{0}$. If this sequence is a convergent sequence, then a limit value of this convergence sequence $c=\lim _{n \rightarrow \infty} x_{n}$ is the fixed point of the function $g$.

If a function $g$ is continuous and the limit value $\lim _{n \rightarrow \infty} x_{n}=c$, then

$$
g(c)=g\left(\lim _{n \rightarrow \infty} x_{n}\right)=\lim _{n \rightarrow \infty} g\left(x_{n}\right)=\lim _{n \rightarrow \infty} x_{n+1}=c .
$$

Hence, the limit value $c$ is a fixed point of the function $g$. This scheme is called as a fixed point iteration method. We only state the theorem without proof. Here, the equation $x_{n+1}=g\left(x_{n}\right)$ is called a iteration equation and the function $g(x)$ is called a iteration function. Also the constant $K$ is the degree of the convergence.

Theorem 2.1 (Fixed Point Iteration). Let $I=[a, b]$ be an interval and let $g(x)$ be a function from $I$ to $I$. Assume that the function $g(x)$ is continuous on $I$ and is differentiable on $(a, b)$. If there exists a constant $0 \leq K<1$ satisfying

$$
\left|g^{\prime}(x)\right| \leq K<1, \quad x \in(a, b) .
$$

Then the sequence $\left\{x_{n}\right\}$ generated by iteration equation

$$
x_{n+1}=g\left(x_{n}\right), \quad n=0,1,2, \cdots
$$

with for any initial guess $x_{0} \in[a, b]$ converges to the fixed point $c$ of the function $g$ on $I=[a, b]$.

In previous theorem, we see that the fixed point iteration is used to way to look for the fixed point of the function $g(x)$, i.e., the roots of the equation $g(x)=x$. In order to find the roots of the equation $f(x)=0$, at first, transformed in the form of $f(x)=0$ to $x=g(x)$. At this $g(x)$ is a function that must be satisfied with condition of the fixed point theorem. That is, if $g(x)$ is a function satisfying $\left|g^{\prime}(x)\right| \leq K<1$ on $I=[a, b]$ for some constant $K$, after we choose an arbitrary initial value $x_{0}$ in the interval $I$, find sequence $\left\{x_{n}\right\}$ to apply $x_{n+1}=g\left(x_{n}\right)$. This sequence converges and limit value of $\left\{x_{n}\right\}$ is fixed point of $g(x)$ i.e. That is a solution of the equation $f(x)=0$. 
Now, let's apply the equation $x^{2}-x-1=0$ to obtain the golden ratio. As seen earlier, we know that one of those roots of this equation is the golden ratio $\phi$. Expressed in the form $x=g(x)$ of the equation $x^{2}-x-$ $1=0$ to obtain this value. Next, make sure that the function $g(x)$ should satisfy the condition of the fixed point iteration in the interval $[a, b]$, where $a>0$. If it satisfies the conditions, by choosing an appropriate initial value $x_{0}$, find a sequence $\left\{x_{n}\right\}$ by applying iteration $x_{n+1}=f\left(x_{n}\right)$. The limit value of the sequence $\left\{x_{n}\right\}$ is a solution of the equation $x^{2}-$ $x-1=0$. This is the golden ratio $\phi$. Now, let's find out the function $g(x)$ by transforming into continued fraction the equation $x^{2}-x-1=0$.

Proposition 2.2. The golen ratio can be represented as following:
(a) $\phi=1+\frac{1}{1+\frac{1}{1+\frac{1}{1+\frac{1}{1}}}}$.
(b) $\phi=2-\frac{1}{3-\frac{1}{3-\frac{1}{3-\frac{1}{2}}}}$.
(c) $\phi=3-\frac{5}{5-\frac{5}{5-\frac{5}{5-\frac{5}{\ddots}}} \text {. }}$
(d) $\phi=2-\frac{1}{2+\frac{1}{2-\frac{1}{2+\frac{1}{\ddots}}} \text {. }}$

Moreover, there may be many other representation of the continued fraction for golden ratio.

Proof. (a) From the equation $x^{2}-x-1=0$, we have $x^{2}=x+1$. Dividing both sides by $x$, then we obtain $x=1+\frac{1}{x}$. Hence we take $g_{1}(x)=1+\frac{1}{x}$. For all $x \in[1.5,2]$,

$$
1.5=1+\frac{1}{2} \leq g_{1}(x)=1+\frac{1}{x} \leq 1+\frac{1}{1.5}<2 .
$$

Hence $g_{1}:[1.5,2] \rightarrow[1.5,2]$ is continuous. Also, $g_{1}$ satisfies $\left|g_{1}^{\prime}(x)\right|=$ $\left|-\frac{1}{x^{2}}\right| \leq \frac{4}{9}<1$. Therefore by the Theorem 2.1, the sequence constructed by the fixed point iteration $x_{n+1}=g_{1}\left(x_{n}\right)$ converges to the fixed point of $g_{1}(x)$ for any initial $x_{0} \in[1.5,2]$. Let $x_{0}=2$ be the initial value, then 
the sequence $\left\{x_{n}\right\}$ is as follows:

$$
\begin{aligned}
& x_{1}=1+\frac{1}{2}=\frac{3}{2} \\
& x_{2}=1+\frac{1}{x_{1}}=1+\frac{1}{1+\frac{1}{2}}=\frac{5}{3} \\
& x_{3}=1+\frac{1}{x_{2}}=1+\frac{1}{1+\frac{1}{1+\frac{1}{2}}}=\frac{8}{5}
\end{aligned}
$$

Hence this sequence $\left\{x_{n}\right\}$ converges to the fixed point of the function $g_{1}(x)$ i.e. the solution of $x^{2}-x-1=0$. Since the limit value exists in the interval $[1.5,2]$,

$$
\phi=\lim _{n \rightarrow \infty} x_{n}=1+\frac{1}{1+\frac{1}{1+\frac{1}{1+\frac{1}{\ddots}}}} .
$$

(b) From the equation $x^{2}-x-1=0$, we have $x^{2}-x-2=-1$.

$$
(x-2)(x+1)=-1 \Longrightarrow x-2=-\frac{1}{x+1} \Longrightarrow x=2-\frac{1}{x+1} .
$$

Hence we take $g_{2}(x)=2-\frac{1}{x+1}$. For all $x \in[1,2]$,

$$
1<\frac{3}{2} \leq g_{2}(x)=2-\frac{1}{x+1} \leq \frac{5}{3}<2 .
$$

Hence $g_{2}:[1,2] \rightarrow[1,2]$ is continuous. Also, $g_{2}$ satisfies $\left|g_{2}^{\prime}(x)\right|=$ $\left|\frac{1}{(x+1)^{2}}\right| \leq \frac{1}{4}<1$. Therefore by the Theorem 2.1, the sequence constructed by the fixed point iteration $x_{n+1}=g_{2}\left(x_{n}\right)$ converges to the fixed point of $g_{2}(x)$ for any initial $x_{0} \in[1,2]$. If initial value is chosen $x_{0}=1$, then the sequence $\left\{x_{n}\right\}$ is as follows:

$$
\begin{aligned}
& x_{1}=2-\frac{1}{1+1}=\frac{3}{2} \\
& x_{2}=2-\frac{1}{1+x_{1}}=2-\frac{1}{1+2-\frac{1}{1+1}}=\frac{8}{5} \\
& x_{3}=2-\frac{1}{1+x_{2}}=2-\frac{1}{1+2-\frac{1}{1+2-\frac{1}{1+1}}}=\frac{21}{13}
\end{aligned}
$$


Hence this sequence $\left\{x_{n}\right\}$ converges to the fixed point of the function $g_{2}(x)$ i.e. the solution of the equation $x^{2}-x-1=0$. Since the limit value exists in the interval $[1,2]$,

$$
\phi=\lim _{n \rightarrow \infty} x_{n}=2-\frac{1}{3-\frac{1}{3-\frac{1}{3-\frac{1}{\ddots}}}} .
$$

(c) From the equation $x^{2}-x-1=0$, we also have $x^{2}-x-6=-5$.

$$
(x-3)(x+2)=-5 \Longrightarrow x-3=-\frac{5}{x+2} \Longrightarrow x=3-\frac{5}{x+2} \text {. }
$$

Hence we take $g_{3}(x)=3-\frac{5}{2+x}$. For all $x \in[1,2]$,

$$
1<\frac{4}{3} \leq g_{3}(x)=3-\frac{5}{2+x} \leq \frac{7}{4}<2 .
$$

Hence $g_{3}:[1,2] \rightarrow[1,2]$ is continuous. Also, $g_{3}$ satisfies $\left|g_{3}^{\prime}(x)\right|=$ $\left|\frac{5}{(2+x)^{2}}\right| \leq \frac{5}{9}<1$. Therefore by the Theorem 2.1, the sequence constructed by the fixed point iteration $x_{n+1}=g_{3}\left(x_{n}\right)$ converges to the fixed point of $g_{3}(x)$ for any initial $x_{0} \in[1,2]$. If initial value is chosen $x_{0}=1$, then the sequence $\left\{x_{n}\right\}$ is as follows:

$$
\begin{aligned}
x_{1} & =3-\frac{5}{2+1}=\frac{4}{3} \\
x_{2} & =3-\frac{5}{2+x_{1}}=3-\frac{5}{2+3-\frac{5}{2+1}}=\frac{3}{2} \\
x_{3} & =3-\frac{5}{2+x_{2}}=3-\frac{5}{2+3-\frac{5}{2+3-\frac{5}{2+1}}}=\frac{11}{7} \\
& \vdots
\end{aligned}
$$

Hence this sequence $\left\{x_{n}\right\}$ converges to the fixed point of the function $g_{3}(x)$ i.e. the solution of the equation $x^{2}-x-1=0$. Since the limit value exists in the interval $[1,2]$,

$$
\phi=\lim _{n \rightarrow \infty} x_{n}=3-\frac{5}{5-\frac{5}{5-\frac{5}{5-\frac{5}{\ddots}}}} .
$$

(d) From the equation $x^{2}-x-1=0$, we also have $2 x^{2}-2 x-2=0$ and $2 x^{2}-3 x-2=-x$.

$$
(x-2)(2 x+1)=-x \Longrightarrow x-2=-\frac{x}{2 x+1} \Longrightarrow x=2-\frac{x}{2 x+1} .
$$


Hence we take $g_{4}(x)=2-\frac{x}{2 x+1}=2-\frac{1}{2+\frac{1}{x}}$. For all $x \in[1,2]$,

$$
1<2-\frac{2}{5} \leq g_{4}(x)=2-\frac{x}{2 x+1} \leq 2-\frac{1}{3}<2 .
$$

Hence $g_{4}:[1,2] \rightarrow[1,2]$ is continuous. Also, $g_{4}$ satisfies $\left|g_{4}^{\prime}(x)\right|=$ $\left|\frac{1}{(2 x+1)^{2}}\right| \leq \frac{1}{9}<1$. Therefore by the Theorem 2.1, the sequence constructed by the fixed point iteration $x_{n+1}=g_{4}\left(x_{n}\right)$ converges to the fixed point of $g_{4}(x)$ for any initial $x_{0} \in[1,2]$. If initial value is chosen $x_{0}=1$, then the sequence $\left\{x_{n}\right\}$ is as follows:

$$
\begin{aligned}
& x_{1}=2-\frac{1}{2+1}=\frac{5}{3} \\
& x_{2}=2-\frac{1}{2+\frac{1}{x_{1}}}=2-\frac{1}{2+\frac{3}{5}}=\frac{21}{13} \\
& x_{3}=2-\frac{1}{2+\frac{1}{x_{2}}}=2-\frac{1}{2+\frac{13}{21}}=\frac{89}{55}
\end{aligned}
$$

Hence this sequence $\left\{x_{n}\right\}$ converges to the fixed point of the function $g_{4}(x)$ i.e. the solution of the equation $x^{2}-x-1=0$. Since the limit value exists in the interval $[1,2]$,

$$
\phi=\lim _{n \rightarrow \infty} x_{n}=2-\frac{1}{2+\frac{1}{2-\frac{1}{2+\frac{1}{\zeta}}}} .
$$

\section{Numerical Computation}

As can be seen in the previous examples, we were able to get the golden ratio represented by continued fraction from functions. By searching for another functions in $g(x)$, can be obtained new representation. The values of these sequences in fact, can be calculated. Let's look at the values of sequence and the number of iteration through numerical calculations. For this computation, we used the MS-EXCEL program.

From the Table 3.2., we know that the less degree $K$ of the convergence makes the faster convergence. Hence we will show the next theorem can be reduce the degree $K$. 


\begin{tabular}{|c|c|c|c|c|}
\hline $\mathrm{n}$ & $g_{1}(x)$ & $g_{2}(x)$ & $g_{3}(x)$ & $g_{4}(x)$ \\
\hline 0 & 2 & 1 & 1 & 1 \\
1 & 1.5 & 1.5 & 1.333333333 & 1.666666667 \\
2 & 1.6666666667 & 1.6 & 1.5 & 1.615384615 \\
3 & 1.6 & 1.615384615 & 1.571428571 & 1.618181818 \\
4 & 1.625 & 1.617647059 & 1.6 & 1.618025751 \\
5 & 1.615384615 & 1.617977528 & 1.611111111 & 1.618034448 \\
6 & 1.619047619 & 1.618025751 & 1.615384615 & 1.618033963 \\
7 & 1.617647059 & 1.618032787 & 1.617021277 & 1.618033990 \\
8 & 1.618181818 & 1.618033813 & 1.617647059 & $\mathbf{1 . 6 1 8 0 3 3 9 8 9}$ \\
9 & 1.617977528 & 1.618033963 & 1.617886179 & $\mathbf{1 . 6 1 8 0 3 3 9 8 9}$ \\
10 & 1.618055556 & 1.618033985 & 1.617977528 & $\mathbf{1 . 6 1 8 0 3 3 9 8 9}$ \\
11 & 1.618025751 & 1.618033988 & 1.618012422 & $\mathbf{1 . 6 1 8 0 3 3 9 8 9}$ \\
12 & 1.618037135 & $\mathbf{1 . 6 1 8 0 3 3 9 8 9}$ & 1.618025751 & $\mathbf{1 . 6 1 8 0 3 3 9 8 9}$ \\
13 & 1.618032787 & $\mathbf{1 . 6 1 8 0 3 3 9 8 9}$ & 1.618030842 & $\mathbf{1 . 6 1 8 0 3 3 9 8 9}$ \\
14 & 1.618034448 & $\mathbf{1 . 6 1 8 0 3 3 9 8 9}$ & 1.618032787 & $\mathbf{1 . 6 1 8 0 3 3 9 8 9}$ \\
15 & 1.618033813 & $\mathbf{1 . 6 1 8 0 3 3 9 8 9}$ & 1.618033530 & $\mathbf{1 . 6 1 8 0 3 3 9 8 9}$ \\
16 & 1.618034056 & $\mathbf{1 . 6 1 8 0 3 3 9 8 9}$ & 1.618033813 & $\mathbf{1 . 6 1 8 0 3 3 9 8 9}$ \\
17 & 1.618033963 & $\mathbf{1 . 6 1 8 0 3 3 9 8 9}$ & 1.618033922 & $\mathbf{1 . 6 1 8 0 3 3 9 8 9}$ \\
18 & 1.618033999 & $\mathbf{1 . 6 1 8 0 3 3 9 8 9}$ & 1.618033963 & $\mathbf{1 . 6 1 8 0 3 3 9 8 9}$ \\
19 & 1.618033985 & $\mathbf{1 . 6 1 8 0 3 3 9 8 9}$ & 1.618033979 & $\mathbf{1 . 6 1 8 0 3 3 9 8 9}$ \\
20 & 1.618033990 & $\mathbf{1 . 6 1 8 0 3 3 9 8 9}$ & 1.618033985 & $\mathbf{1 . 6 1 8 0 3 3 9 8 9}$ \\
21 & 1.618033988 & $\mathbf{1 . 6 1 8 0 3 3 9 8 9}$ & 1.618033987 & $\mathbf{1 . 6 1 8 0 3 3 9 8 9}$ \\
22 & $\mathbf{1 . 6 1 8 0 3 3 9 8 9}$ & $\mathbf{1 . 6 1 8 0 3 3 9 8 9}$ & 1.618033988 & $\mathbf{1 . 6 1 8 0 3 3 9 8 9}$ \\
23 & $\mathbf{1 . 6 1 8 0 3 3 9 8 9}$ & $\mathbf{1 . 6 1 8 0 3 3 9 8 9}$ & $\mathbf{1 . 6 1 8 0 3 3 9 8 9}$ & $\mathbf{1 . 6 1 8 0 3 3 9 8 9}$ \\
24 & $\mathbf{1 . 6 1 8 0 3 3 9 8 9}$ & $\mathbf{1 . 6 1 8 0 3 3 9 8 9}$ & $\mathbf{1 . 6 1 8 0 3 3 9 8 9}$ & $\mathbf{1 . 6 1 8 0 3 3 9 8 9}$ \\
25 & $\mathbf{1 . 6 1 8 0 3 3 9 8 9}$ & $\mathbf{1 . 6 1 8 0 3 3 9 8 9}$ & $\mathbf{1 . 6 1 8 0 3 3 9 8 9}$ & $\mathbf{1 . 6 1 8 0 3 3 9 8 9}$ \\
\hline
\end{tabular}

TABLE 3.1. The values of sequences for each iteration function

\begin{tabular}{|c|c|c|c|c|}
\hline $\mathrm{n}$ & $g_{1}(x)$ & $g_{2}(x)$ & $g_{3}(x)$ & $g_{4}(x)$ \\
\hline the number of iteration & 22 & 12 & 23 & 8 \\
\hline degree of the convergence & $\frac{4}{9}$ & $\frac{1}{4}$ & $\frac{5}{9}$ & $\frac{1}{9}$ \\
\hline
\end{tabular}

TABLE 3.2. The comparison of the number of iteration with the degree of the convergence $K$

Theorem 3.1. Assume that the functions $g_{1}$ and $g_{2}$ are the iteration function in the Theorem 2.1 with the degree of the convergence $K_{1}$ and $K_{2}$ and that two functions have the same fixed point $c$. Then we have 
another iteration function $g_{1} \circ g_{2}$ which satisfies the condition in Theorem 2.1 with the degree of the convergence $K_{1} K_{2}$ which is less than $K_{1}$ and $K_{2}$. In other words, the equation $\left\{x_{n}\right\}$ generated by iteration equation

$$
x_{n+1}=g_{1} \circ g_{2}\left(x_{n}\right)=g_{1}\left(g_{2}\left(x_{n}\right)\right), \quad n=0,1,2, \cdots
$$

with for any initial guess $x_{0}$ converges to the same fixed point $c$.

Proof. Since $g_{1}$ and $g_{2}$ are the iteration function with the degree $K_{1}$ and $K_{2}$, respectively, i.e.,

$$
\left|g_{1}^{\prime}(x)\right| \leq K_{1}, \quad\left|g_{2}^{\prime}(x)\right| \leq K_{2}, \quad x \in I,
$$

then

$\left|\left(g_{1} \circ g_{2}\right)^{\prime}(x)\right|=\left|g_{1}^{\prime}\left(g_{2}(x)\right) \cdot g_{2}^{\prime}(x)\right|=\left|g_{1}^{\prime}\left(g_{2}(x)\right)\right| \cdot\left|g_{2}^{\prime}(x)\right| \leq K_{1} K_{2}, \quad x \in I$.

Hence $g_{1} \circ g_{2}$ is iteration function with the degree $K_{1} K_{2}$. By Theorem 2.1., the sequence $\left\{x_{n}\right\}$ generated by the iteration function $g_{1} \circ g_{2}$ converges to the fixed point of $g_{1} \circ g_{2}$.

Since $g_{1}$ and $g_{2}$ have the same fixed point $c$, i.e., $g_{1}(c)=c$ and $g_{2}(c)=c$, then

$$
\left(g_{1} \circ g_{2}\right)(c)=g_{1}\left(g_{2}(c)\right)=g_{1}(c)=c .
$$

Corollary 3.2. If $g(x)$ is a iteration function, then the composite function $g \circ \cdots \circ g$ is also iteration function.

By Proposition 2.2. (a), $g_{1}(x)=1+\frac{1}{x}$ is the iteration function with $K_{1}=\frac{4}{9}<1$. By Corollary $3.2, g_{1} \circ g_{1}$ is also the iteration function for the same fixed point. It is easy to check that the function $g_{1} \circ g_{1}$ is equal to the function $g_{2}$ in Proposition 2.2. (b).

By Corollary 3.2., $g_{1} \circ g_{1} \circ \cdots \circ g_{1}=1+\frac{1}{1+\frac{1}{1+\frac{1}{\ddots}}}$ is the iteration

$$
\ddots+\frac{1}{x}
$$

function with $K=\left(\frac{4}{9}\right)^{n}$ as $n$ tends to $\infty$, the limit function $g=\lim \left(g_{1}\right)^{n}$ is the iteration function and satisfies

$$
\left|g^{\prime}(x)\right|=\lim \left|\left(g_{1}^{\prime}\right)^{n}\right| \leq \lim K_{1}^{n}=0 .
$$

Hence $g(x)$ is a constant function with fixed point $c$. Therefore

$$
\lim _{n \rightarrow \infty} g_{1} \circ g_{1} \circ \cdots \circ g_{1}=1+\frac{1}{1+\frac{1}{1+\frac{1}{1+\frac{1}{\ddots}}}}=\phi .
$$




\section{Concluding Remarks}

We investigated the definition and expression of the golden ratio, and inquired about the required courses for the golden ratio is represented by continued fraction, fixed point, sequence, fixed point iteration, by considering the mathematical theory for only known contents as the numerical value. Through this process, we could understand for relationship between the golden ratio and continued fraction. Also we were able to visualize the result through numerical calculations using the MSEXCEL program.

\section{References}

[1] R. G. Bartle and D. R. Sherbert, Introduction to Real Analysis, 3rd Ed., JOHN WILEY \& SON, 2000.

[2] James F. Epperson, An Introduction to Numerical Methods and Analysis, JOHN WILEY \& SON, INC 2002.

[3] A. Y. Khinchin, Continued fractions, Dover Publications, inc., 1997.

[4] S. L. Griffing, The Golden Section : An Ancient Egyptian and Grecian Proportion, Xlibris Corporation, 1998.

Seung Soo Kim

Department of Mathematics (Institute of Pure and Applied Mathematics), Chonbuk National University,

Jeonju, 561-756, Republic of Korea.

E-mail : prettyss@jbnu.ac.kr

Mi Yeon Ko

Department of Mathematics Educations, Graduate School, Chonbuk National University, Jeonju, 561-756, Republic of Korea.

Yong Hun Lee

Department of Mathematics (Institute of Pure and Applied Mathematics), Chonbuk National University,

Jeonju, 561-756, Republic of Korea.

E-mail : lyh229@jbnu.ac.kr 